\title{
Case Report: Health Services Vulnerability During the Ebola Outbreak: A Qualitative Report
}

\author{
Nasir Amanat ${ }^{1}$, Seyed Hossein Hosseini ${ }^{1 *}$, Hamid Reza Khankeh ${ }^{1,2}$, Mohsen Aminizadeh ${ }^{1}$, Maryam Nakhaee ${ }^{3}$, Babak Farzinnia \\ 1. Research Center in Emergency and Disaster Health, University of Social Welfare and Rehabilitation Sciences, Tehran, Iran. \\ 2. Department of Clinical Science and Education, Karolinska Institute, Stockholm, Sweden. \\ 3. Social Determinants of Health Research Center, Birjand University of Medical Sciences, Birjand, Iran.
}

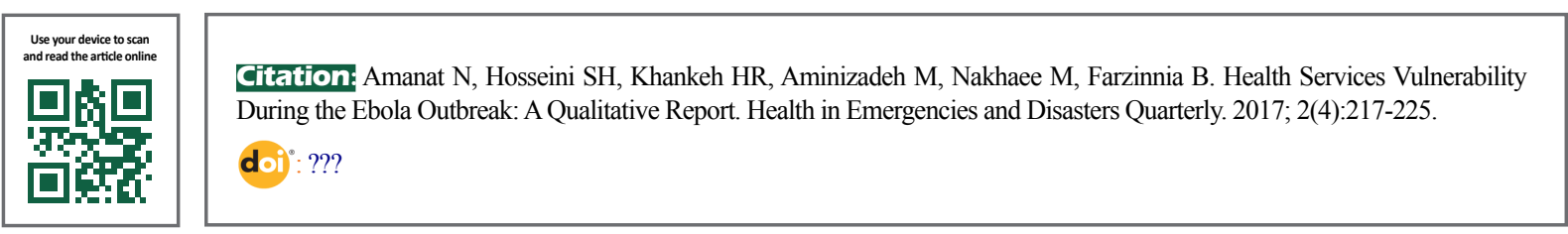

Article info:

Received: 12 Jan. 2017

Accepted: 28 Apr. 2017

\section{Keywords:}

Ebola, Injury, Health personnel, Qualitative case study

\section{ABSTRACT}

Ebola is an infectious disease, which is caused by a virus belonging to the Filoviridae group. The outbreak of the disease in the African countries in 2015 caused massive death and contamination of the healthcare personnel those who were engaged in treating the infected patients and caused irreparable damage to the healthcare system. In this study, the vulnerability of the team of health service providers during the Ebola outbreak in Liberia, Guinea and Sierra Leone is studied. The article also proposes solutions that can be learned as a lesson, help in increasing their resilience in similar biological hazards and planning management strategies for similar events in the future. Long before the outbreak took place, West African countries were already facing acute problems in terms of access to health services and health infrastructure. The most important shortcomings for the same were identified as insufficient number of health personnel and capacity shortage that prevented the people from being ready to deal with such uncalled events viz. accidents and epidemic disease outbreak. The Ebola epidemic exacerbated the persisting problems caused due to a shortage of personnel in these countries and caused the death of a large number of common people as well as healthcare personnel. Generally, the vulnerability of the health team working during the Ebola outbreak could be divided into five general dimensions: 1. Management weakness; 2 . Lack of engineering and environmental control; 3. Obstacles in the use of personal protective equipment; 4. Not having enough skills and practice exercises; and 5. Ignoring the social factors and satisfaction of the healthcare personnel. The main theme of the study was failure to understand the risk of personnel in accidents and disasters. Findings revealed building capacity and reducing vulnerability of the healthcare personnel against disasters and epidemics depends upon the perceived risk, which is a decisive factor for any intervention. Maintenance of human resources is impossible unless with the sole aim of promoting resilience in various areas of management, health, environmental control, the proper use of personal protective equipment, teaching training, skill upgrading of personnel, and increased social and material support are achieved.

\footnotetext{
* Corresponding Author: 


\section{Introduction}

bola is an infectious disease which is caused by a virus belonging to the Filoviridae cate$\mathbf{E}$ gory. The virus transmits from infected human beings to healthy ones through close contact with either the secretions of the affected person or the dead bodies of the persons who succumbed to Ebola infection or the infected environment. If infected, the patients start showing severe and uncontrollable symptoms such as nausea, diarrhea, and bleeding [1]. Ebola epidemics can affect any country and cause undesirable consequences both in terms of human lives and economic losses. It is therefore recommended that each country should be prepared for the worst. The recent Ebola outbreak in West Africa showed that absence of a resilient health system that is prepared to tackle such epidemic events can create fundamental problems in maintaining the community health of the country [2].

In a general context, a resilient system is prepared to absorb energy from a hazard while providing the basic health services at the same time [3]. Therefore, the presence of a resilient health system is crucial to the medical infrastructure of any nation. Such a system has direct effects on the resilience of the community and helps in increasing the level of readiness to mitigate the effects of disasters [2]. One of the most important aspects of the health system's resilience is the existence of enough personnel who are trained enough to handle such circumstances [3]. That is why protecting health personnel is essential to build capacity and to respond effectively to such incidents. Hence, maintaining and supporting the health of such healthcare personnel should be considered as the main axis in the various phases of disaster management cycle, including readiness, response, and resilience of the system as well as a priority in health system management [3].

Thus, identifying the factors responsible for the vulnerability of these personnel and, trying to fix them can play important roles in promoting the resilience of the health system and society. In the last 40 years, more than 20 Ebola outbreaks have been reported in the African countries, including the outbreaks in Zaire and Sudan in 1976 and Congo in 1995 [4, 5]. The rampant spread of the disease in West Africa started in 2013 and it aggravated to a such a degree that after a period of only two years, February 2015 to be more specific, 22495 (definite, probable, suspicious) Ebola cases and 8981 Ebola associated deaths with mortality rates of $40 \%$ in 9 countries of Guinea, Liberia, Mali, Nigeria, Senegal, Sierra Leone, Spain, England and the United States were recorded.

However, the highest severity of disease was observed in the three countries of Guinea, Liberia and Sierra Leone, where around 10500 people were infected by the virus. Such medical emergency had destructive and severe economic, mental and psychological effects on the socio-economic structure of these countries [6]. In fact, the Ebola outbreak challenged the health system's ability of these affected countries in such a way that in 2014 World Health Organization had to announce Public Health Emergency of International Concern (PHEIC) in West Africa [7, 8].

Even though the importance of the subject has already been realized, very few studies worked on the vulnerability of health personnel engaged in managing the patients during the Ebola outbreak. However, these studies sufficiently highlighted the importance of studying causes and ways of vulnerability, especially in the three countries listed with high transfer volume and how it affected the health care population affected by the virus [9-11].

Case study of health and treatment systems, which faced with such dangers provides further understanding and contributes to the development of the concept of vulnerability. These factors may further be applied for devising ways that can improve the resilience of the health system. The qualitative case study described in this article was conducted with the goal of reporting health problems and vulnerabilities during the Ebola outbreak in these three African countries viz. Guinea, Liberia and Sierra Leone. It was also intended to provide recommendations and strategies to increase the resilience of health personnel in similar viral outbreaks.

\section{Case Report}

Even before the Ebola outbreak, West African countries have been identified to be in poor condition in terms of access to health services and health infrastructure, such as safe drinking water [7]. The expensive cost that people paid for receiving health care services increased poverty, and created financial class distances in these countries, which further degraded the health care conditions. Many healthcare centers were short staffed and unable to provide the necessary services. This situation shows the lack of capacity building in these countries to manage such massive epidemic outbreaks of diseases. During the Ebola outbreak, these affected countries had the lowest capacity to establish a health care system to tackle the increasing number of laboratory tests, and control the 


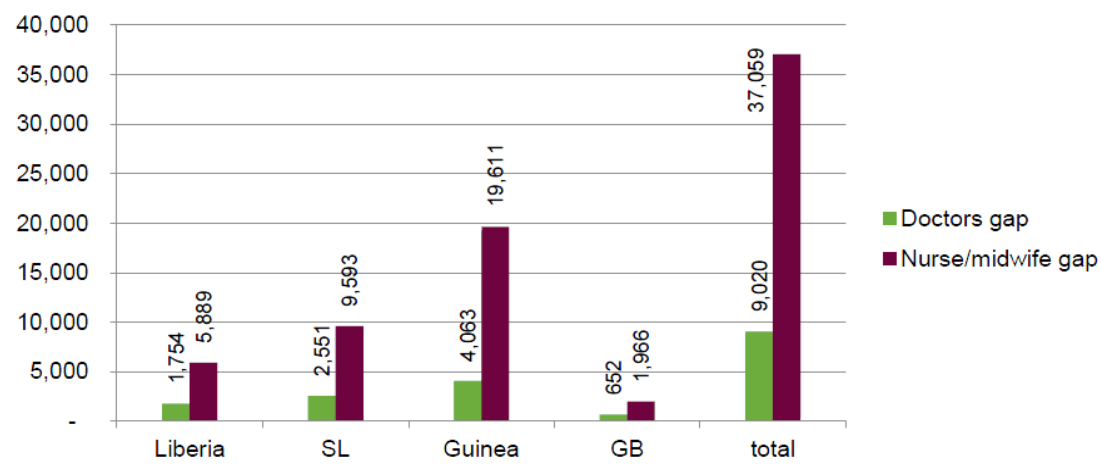

Note: Estimates by Oxfam, based on the WHO minimum standard of 2.3 doctors, nurses and midwives per 1,000 persons. See Annex 1 for calculations.

Figure 1. The shortage of doctors, nurses, and midwives in the four African countries, according to Oxfam report

spread of infection. Fear and distrust in the health system further led to the delay in initiating and implementing effective and efficient response as was needed [12].

The situations thus emerged revealed important and fundamental problems in the healthcare system of these countries, such as persisting discrepancies in the role of the society as a whole in protecting and promoting health, the role of healthcare personnel in providing the appropriate health care services required for controlling the spread of infection, accurate publication of medical information as well as establishing a health care and health promotion system. According to the WHO standards, the number of healthcare staff trained in countries must be 2/3 physician, nurse and midwife per 1000 people.

According to Oxfam (Figure 1), the number of people in these three countries faced with a lot of deficiencies, the fulfillment of which will cost $\$ 420$ million to train 90020 physicians, 37059 nurses and midwives and fill this shortage of manpower (Figure 2). Furthermore, a severe shortage of health centers and hospitals in these countries was also recorded (Figure 3) [3]. The Ebola epidemic further exacerbated the shortage of personnel in these countries, especially because of the fact that since March 1, 2015, a total of 492 health personnel died [7]. Since healthcare personnel have a key role in preventing the spread of Ebola by treating and tending to the needs of the already infected patients, it is natural to presume that the risk of contact with patients and contaminated discharge was very high [4].

As in Sierra Leone, the occurrence of Ebola in health personnel was 100 times more than ordinary people (Incidence rate of 80.04 per 100,000 people) [9]. High incidence of this disease in these people indicates their high vulnerability to the disease. It was also seen that 318 healthcare staff were infected in the three above mentioned countries in the first 9 months of the outbreak, out of which 152 people died (mortality rate of 48\%). By February 2015, the number of infected rose to 822 and so did the mortality rate (488 died, i.e. mortality rate of 59\%) [13].

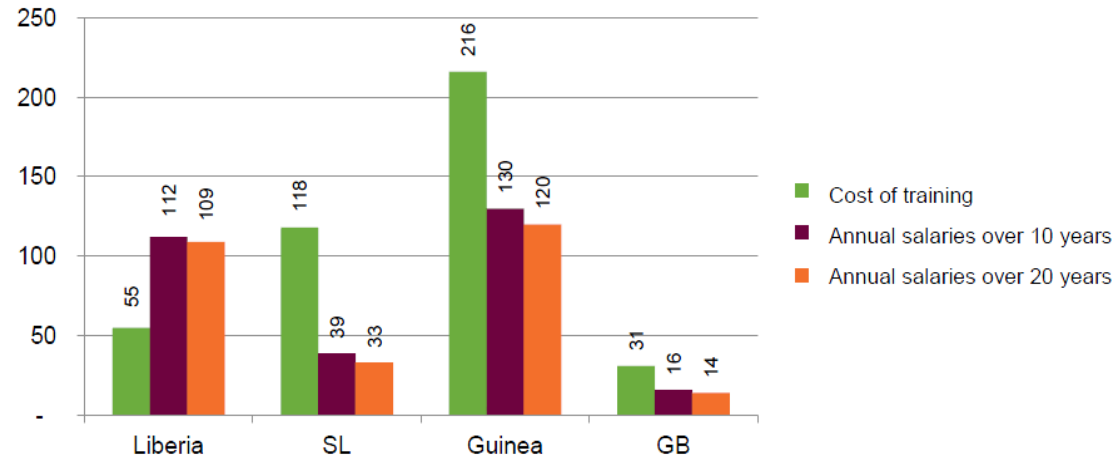

Note: Estimated by Oxfam; see Annex for methodology

IHealth in

Emergencies and |Disasters [Oluarterly

Figure 2. The cost needed for training and salary of health care personnel in four African countries 


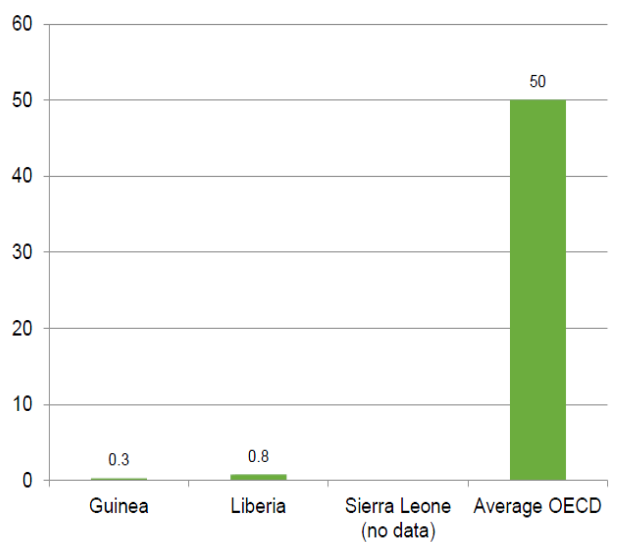

Figure 3. Number of hospitals in the three African countries per 1000 population

Hlealth in

Emergencies and |D]isasters [Oluarterly
This outbreak had very devastating effects on the fragile and vulnerable health systems of these countries and especially its healthcare personnel, among which nurses and nurse assistants made up more than $50 \%$ of those infected with the virus. However, reduction of the percentage of healthcare personnel infection from $12 \%$ in July 2014 to less than 1\% in February 2015 as a result of working in close cooperation with WHO and the Ministry of Health of these countries and use the strategy of maintaining health and Professional Security 0HS, founding guidelines for the prevention and protection of infectious diseases sowed that the infection of the health team and the outbreak is preventable with reduced vulnerability and increasing their resilience [8].

\section{Materials and Methods}

The present study presents a qualitative case report, which was studied through the content analysis approach. At first, reports by internationally recognized organizations, such as Oxfam, WHO, presentations and interviews in the context of the Ebola outbreak were collected from the three countries of Guinea, Liberia and Sierra Leone. The qualitative content analysis approach was used to analyze them on the basis of the Graneheim method [14]. For content analysis, the collected resources were read carefully several times and parts related to the research question were initially encoded and then the same codes were sorted and categorized into subcategories, main categories and themes.

According to the analysis of the quality content of Graneheim and Lundmann, qualitative content analysis was done with varying degrees of interpretations and concentrations. In any text or image, there are obvious messages for description and latent meaning for interpretation. Both obvious and latent messages need interpretation, some of which may vary in depth and abstraction levels [15]. In Table 1, the vulnerability of health personnel during the Ebola outbreak in the three countries of Liberia, Guinea and Sierra Leone are categorized in different areas such as failure to understand and conceive the risk during care.

The main theme of the study was the role of failure to understand the risk healthcare personnel are exposed to while treating patients because of accidents and disasters. According to the findings of the study, failure to understand risk promotes risky behaviors and increases vulnerabilities on numerous occasions. Among the examples of this lack of recognition are the risk of contact with bodies, failure to comply with the standards and precautions for dealing with an infected patient (when dealing with blood and secretions), failure to take medical history of patients and re-examine them to understand the new symptoms of Ebola (especially people under 5 years old) and delay in laboratory diagnosis. In this study, five main categories were formed, which included:

\section{Management weakness}

Occupational Safety and Health Administration (OSHA), National Fire Protection Association (NFPA), many government agencies and trade associations have standards for protecting people at work. One of these standards is management controls. Ignoring these standards can have irreparable effects on the staff. This category can be further categorized into the following:

\section{Patient traffic and transmission problems}

The cases reported in this section include not performing triage or performing triage in the wrong way, inappropriate control of patients and health personnel while moving between health centers. 
Table 1. Vulnerability indicators of health services providers in the Ebola outbreak

\begin{tabular}{ll}
\hline \multicolumn{2}{l}{ Lack of Understanding and Realizing the Risk During Care (Main Themes) } \\
\hline Categories & Sub-Categories \\
\hline
\end{tabular}

Failure to comply with the control instructions and prevention of infectious diseases

Management weakness

Lack of engineering and environmental control

Obstacles to the use of personal protective equipment

Not having enough skills and operational exercises

Ignoring social factors and staff satisfaction
Problems with entering, leaving and carrying patients

Lack of equipment and personnel

Lack of proper isolation

Lack of environmental control

Failure to use or incorrect use of PPE

Non permanent use of PPE

Not having skills and not practicing hand washing

Lack of skill in collecting biological samples

No or delayed payment of rewards and fees

Lack of societal supports

Emergencies and D]isasters [O]uarterly

Failure to comply with the control instructions and Infectious Prevention Diseases (IPC)

One of the most important vulnerability factors of the Ebola epidemic was the lack of standard operating procedures and specific responsibilities in establishing the IPC and lack of expertise in IPC.

\section{Absence of equipment and personnel}

Cases of capacity shortages rely on the lack of equipment, tools, proper training, limited capacity or wrong instruction in the field of managing contaminated equipment, lack of required capacity or proper education in the management of corpses.

\section{Lack of engineering and environmental control}

The second principle of the US Occupational Health and Safety Administration is engineering control. Among the topics neglected in these countries are:

\section{Lack of proper isolation}

Lack of perception of danger at different levels in the health system and the weakness of the infrastructure caused the risks of not being investigated. Also, there were no programs that meant for making preparations and capacity building. Among the examples are proper facilities for isolation, not separating high-risk areas and low-risk areas, infrastructure constraints, not separating public sectors from Ebola patients and lack of access to proper transportation system for patients and corpses.

\section{Non-environmental control}

This part includes improper decontamination of equipment and surfaces, lack of adherence to the principles of hand hygiene and lack of using soap and alcohol.

Barriers to the use of Personal Protective Equipment (PPE)

OSHA regulations require PPE of eyes, face, head, and body while performing dangerous actions, including while handling patients suffering from dangerous contagious diseases. Other considerations include hearing protection, protective clothing, protective coatings, barriers and respiratory equipment. Hazards may include process or environmental hazards, chemical hazards, radiological hazards or mechanical stimulants which may involve individuals through absorption, inhalation or physical contact with chemicals. In the spring of 1994, the OSHA revised its PPE regulations and assessed the risk to determine the need to use PPE for preventing possible damage [16]. It was identified that there were many barriers to using PPE during Ebola outbreak including lack of use or improper use of PPE, non-permanent use 
of PPE, multiple use of already used PPE and personnel resilience in using PPE.

\section{Not enough skills and operational exercises}

Analyzing the problems faced during this outbreak also indicated lack of exercise and low staffing skill. Lack of skills and not practicing hand washing, lack of skill in safe methods of collecting biological samples, the injuries caused by needle stick, having contact with mucus while removing PPE, smoking when wearing PPE, using mobile phone, nursing care at home and hugging colleagues are the most important factors under this domain. On the basis of the curriculum, health system preparedness as well as training and practice of the components of the health team's readiness [17] and preparing an effective response to an urgent urgency and to acquire or upgrade basic skills and competencies along with multi-year planning was recommended [18].

\section{Overlooking the social factors and satisfaction of personnel}

Overlooking the social factors and satisfaction of personnel includes absence or delay in payment of rewards and fees, lack of social support [13].

\section{Discussion}

\section{Lack of understanding the danger}

Vulnerability of health system personnel during the Ebola outbreak in the three countries was categorized into main theme, five categories, and eleven subcategories. The main theme of the study was the "lack of understanding of risk". Health and safety principles are based on possible workplace hazards. Known working conditions are not likely to change every day; the staff members perform the same tasks, and in general, they have an implicit understanding of it. However, conditions during emergency operations can vary greatly. In emergencies, most likely, risks are not fully understood, especially in the initial response operation, risks may vary sharply throughout the response operation [14].

The importance of understanding the risk is so great that it is emphasized as the first priority of the Sendai Framework. This framework is an international road map for countries. The main categories include management weakness, lack of engineering and environmental control, obstacles to the use of personal protective equipment, not enough skills and operational exercises and ignoring social factors. The countries present at the meeting were required to provide practical solutions to increase the perception of danger in different categories, including people and authorities [15].

\section{Management weakness}

Management weakness category includes three subcategories: failure to comply with the control instructions and prevention of infectious diseases; problems arising from admission, discharge, and carrying patients, and the lack of equipment and personnel. Regarding "observing the control instructions and prevention of infectious diseases", development of IPC training is considered as the main factor in the capacity building of the healthcare personnel which in turn will lead to effective risk management in health centers [17]. Also, important topics which are always emphasized in the management of events, having programs at different levels and based on risk. In this program, standard protocols and procedures, roles and responsibilities should be determined [18].

Campbell et al. Miller and WHO reports, all emphasized the importance of complying with the control and prevention guidelines $[19,20]$. Other cases of personnel vulnerability include "problems due to traffic and transfer and patient triage in the health center". It is as one of the most important categories in the management of accidents in the health system, especially hospitals, management of injuries at admission, and intra-hospital and inter-hospital transfers. For this reason, the specialized functions of any hospital include function of triage, decontamination, and having operational plans and protocols in the nearby regions [16].

The existence of this vulnerability suggests the absence of program, practice, and acquiring the necessary skills in this regard. Availability of equipment and personnel in health centers is necessary in capacity management and capacity building which is an important factor for readiness of healthcare personnel and centers [16]. In fact, capacity building is imminent for effective response at reasonable times and times of emergency. In a study by Margaret et al. and the Oxfam Institute it was found that capacity building in the field of personnel and equipment play important roles in the health system's resilience $[3,21]$. Of course, in this study, the lack of capacity building and the shortage of personnel are mentioned as the employee vulnerability in the health system that should be paid attention.

\section{Engineering and environmental control}

Another major category in this study is "Engineering and Environmental Control", which has two sub-catego- 
ries viz. "lack of isolation" and "environmental control". Multiple studies also mentioned availability of specific places such as isolated or quarantined parts to prevent the spread of disease and the effects of healthy people as one of the resilience aspects of self-regulation [19-21]. In addition, it seems that in this case, lack of risk perception at different levels in the health system and the weakness of the infrastructure caused lack of having a program for creating readiness and capacity building.

Obstacles to the use of personal protective equipment

The other main category is "Obstacles to using personal protective equipment" which has two sub-categories viz. "lack of using PPE" and "non-persistent use of PPE". The study of William et al. is in congruence with this study and indicated that using PPE for personnel during Ebola outbreak is considered necessary but insufficient and they acknowledged that either pulling out or removing PPE due to sweating can also lead to contamination of membranes in warm and humid conditions. In addition, fatigue increases the chance of error [22]. Lesperance also mentioned it as one of the factors of the resilience of the health team, and indicated that wearing PPE provides relaxed and secure feelings in the personnel, especially during epidemics [20].

\section{Not having sufficient training and training}

Another main category is "lack of enough training and training" with two sub-categories viz. "lack of skills and not practicing hand washing" and "lack of skill in collecting biological samples". It should also be considered that respondents who deal with such biological agents should be appropriately trained and informed about the possible risks that they will be facing in such grave conditions. Training on operation time is required initially and annually. Training should be conducted both in classroom and at work with a qualified trainer and the records of education should be preserved. When a new hazard is created, retraining is required [14].

Furthermore, training must be followed by a practicing stage, which will ensure the real time readiness of the personnel [21]. Exercises can be discussion-based or operation-based, which ultimately cause finding weaknesses in the program and increasing skill in procedures [21]. Previous studies have identified that weakness of the training, lack of training, and lack of educational curriculum are critically emphasized in the in-service training regimen but training during education is usually generalized and non-specific $[17,22]$.
Ignoring social capital and satisfaction of the health team

The last main category is "Ignoring social capital and satisfaction of the health team" was further divided into two sub-categories viz. "lack or delayed payment of rewards and payments" and "lack of social protection". Its effects can be shown as weakening of cooperation, no motivation, and eventually reduced productivity. In health system, social capital has two dimensions: sense of value and sense of responsibility among the staff and a comprehensive and strong community engagement with the health sector. Strengthening this capital, especially in the pre-incident stage, promotes the psychological resilience of personnel after enlarged traumas and speeds up the recovery of the health system.

Paying attention to personnel motivational factors in many ways can be effective in effectuating the success of healthcare programs [23]. In line with this study, in the study of Shine et al. too indicated that social capital is the most important dimension of community rejuvenation during and after the Ebola outbreak [24]. This is justified by the fact that the health system and its medical staff are actually representative of the community.

\section{Conclusion}

Considering the above propositions and discussion and lessons learned from the Ebola outbreak in African countries, it was concluded that the following points should be addressed on an immediate basis so as to increase the resilience of the healthcare team in accidents and disasters, especially in epidemic disease outbreaks: paying attention to the first priority of the Sendai framework i.e. to understand the danger of disasters and considering that to modify the methods and policies of healthcare management. It was also proposed that doing so will play a significant role in reducing vulnerability and taking risk reduction measures in the healthcare system. Therefore, it is recommended that policy makers and health managers should place these points in their planning priorities.

Furthermore, it must also be considered that since work force is the most important capital of a system and organization [23], reduced human resource vulnerability and their increased ability will play essential role in effective and timely response to disasters in the healthcare system too. Moreover, the protection and support of health personnel and their increased resilience must be given due importance in planning and preparation of response stages and building a resilient health system. Countries which are already identified to have fragile and vulnerable health 
infrastructure in different dimensions, including the human resources sector, need to initiate long-term strategic planning to increase capacity and reduce vulnerability.

In addition, implementing comprehensive programs that will augment the process of acquiring and practicing recommended skills, as well as preparing for performing procedures, paying attention to the materialistic and spiritual aspects of the personnel, increasing satisfaction, and implementing motivational power play can help in successful planning and preparation of the personnel for accidents and disaster management programs. Thus, with resilient personnel, resilient health system can be reached and from resilient health system, resilient society can be reached.

\section{Acknowledgments}

This research did not receive any specific grant from funding agencies in the public, commercial, or not-forprofit sectors. The authors express their thanks and gratitude to the head of the health research center in disasters of University of Rehabilitation Sciences and Social Welfare for their support.

\section{Conflict of Interest}

The authors declared no conflicts of interest.

\section{References}

[1] Dowell SF, Mukunu R, Ksiazek TG, Khan AS, Rollin PE, Peters CJ. Transmission of Ebola hemorrhagic fever: a study of risk factors in family members, Kikwit, Democratic Republic of the Congo, 1995. Journal of infectious diseases. 1999; 179(Supplement 1):S87-S91. doi: 10.1086/514284

[2] Kieny MP, Evans DB, Schmets G, Kadandale S. Health-system resilience: Reflections on the Ebola crisis in western Africa. Bulletin of the World Health Organizatio. 2014; 92(12):850. doi: 10.2471/blt.14.149278

[3] Kamal Yanni M. Never again: Building resilient health systems and learning from the Ebola crisis. Oxford: Oxfam; 2015.

[4] Hewlett BL, Hewlett BS. Providing care and facing death: Nursing during Ebola outbreaks in central Africa. Journal of Transcultural Nursing. 2005; 16(4):289-97. doi: $10.1177 / 1043659605278935$

[5] Bres P. The epidemic of Ebola haemorrhagic fever in Sudan and Zaire, 1976: Introductory note. Bulletin of the World Health Organization. 1978; 56(2):245. PMCID: PMC2395566
[6] World Health Organization. WHO statement on the $1^{\text {st }}$ meeting of the international health regulations emergency committee regarding the 2014 Ebola outbreak in West Africa. Geneva: World Health Organization; 2014.

[7] Corsi JR. 1.2 million Ebola deaths projected in 6 months. Pennsylvania: World Net Daily; 2014

[8] Kilmarx PH, Clarke KR, Dietz PM, Hamel MJ, Husain F, McFadden JD, et al. Ebola virus disease in health care workers Sierra Leone, 2014. Morbidity and Mortality Weekly Report. 2014; 63(49):1168-71. PMID: 25503921

[9] Matanock A, Arwady MA, Ayscue P, Forrester JD, Gaddis B, Hunter JC, et al. Ebola virus disease cases among health care workers not working in Ebola treatment units-Liberia, June-August, 2014. Morbidity and Mortality Weekly Report. 2014; 63(46):1077-81.

[10] Forrester JD, Hunter JC, Pillai SK, Arwady MA, Ayscue P, Matanock A, et al. Cluster of Ebola cases among Liberian and US health care workers in an Ebola treatment unit and adjacent hospital-Liberia, 2014. Morbidity and Mortality Weekly Report. 2014; 63(41):925-9. PMID: 25321070

[11] Women UN. Ebola outbreak takes its toll on women. New York: UN Women News. 2014.

[12] Milland M, Bolkan HA. Enhancing access to emergency obstetric care through surgical task shifting in Sierra Leone: Confrontation with Ebola during recovery from civil war. Acta Obstetricia et Gynecologica Scandinavica. 2014; 94(1):57. doi: $10.1111 /$ aogs.12540

[13] World Health Organization. Health worker Ebola infections in Guinea, Liberia and Sierra Leone: A preliminary report 21 May 2015. Geneva: World Health Organization; 2015.

[14] Graneheim U, Lundman B. Qualitative content analysis in nursing research: Concepts, procedures and measures to achieve trustworthiness. Nurse Education Today. 2004; 24(2):105-12. doi: 10.1016/j.nedt.2003.10.001

[15] Egberg Thyme K, Wiberg B, Lundman B, Graneheim UH Qualitative content analysis in art psychotherapy research: Concepts, procedures, and measures to reveal the latent meaning in pictures and the words attached to the pictures. The Arts in Psychotherapy. 2013; 40(1):101-7. doi: 10.1016/j. aip.2012.11.007

[16] Fagel MJ. Principles of emergency management and Emergency Operations Centers (EOC). Boca Raton, Florida: CRC Press; 2016. doi: 10.1201/b10405

[17] Ardalan AM, Saberinia A. [IR Iran national health disaster and emergency response operation plan (Persian). Tehran: Azarbarzin. 2015.

[18] United States Department of Homeland Security. Homeland security exercise and evaluation program (HSEEP). Washington, D.C.: United States Department of Homeland Security; 2013.

[19] Campbell J, Cometto G, Rasanathan K, Kelley E, Syed S, Zurn $\mathrm{P}$, et al. Improving the resilience and workforce of health systems for women's, children's, and adolescents' health. BMJ. 2015; h4148. doi: 10.1136/bmj.h4148

[20] Lesperance AM, Miller JS. Preventing absenteeism and promoting resilience among health care workers in biological 
emergencies. Richland, WA: US Department of Energy, Pacific Northwest National Laboratory; 2009.

[21] Kruk ME, Myers M, Varpilah ST, Dahn BT. What is a resilient health system? Lessons from Ebola. Lancet. 2015; 385(9980):1910-2. doi: 10.1016/s0140-6736(15)60755-3

[22] Fischer WA, Hynes NA, Perl TM. Protecting health care workers from Ebola: Personal protective equipment is critical but is not enough. Annals of Internal Medicine. 2014; 161(10):753. doi: $10.7326 / \mathrm{m} 14-1953$

[23] Rezaian A. [Fundamentals of organization and management (Persian)]. Tehran: SAMT; 2013.

[24] Michel S, Megerdoomian K. Modeling community resilience for a post-epidemic society. Paper presented at the Computational Social Science Society of the Americas. 2015 October $29^{\text {th }}-$ November $1^{\text {st. }}$ Santa Fe, New Mexico. 